Examining the Efficacy of an Online Program to Cultivate Mindfulness and SelfCompassion Skills (Mind-OP): Randomized Controlled Trial on Amazon's Mechanical

\title{
Turk
}

\author{
Shadi Beshai, Ph.D.*1 \\ University of Regina \\ Christine Bueno, B.A.1 \\ University of Regina \\ Mabel Yu, M.A.1 \\ University of Regina \\ Justin R. Feeney, Ph.D.2 \\ University of Regina \\ Adrian Pitariu, Ph.D.2 \\ University of Regina
}

1. Department of Psychology, University of Regina, 3737 Wascana Parkway, Regina, SK. S4S0A2.

2. Faculty of Business Administration, University of Regina, 3737 Wascana Parkway, Regina, SK. S4S0A2.

* Corresponding Author: Shadi Beshai, Ph.D., shadi.beshai@ uregina.ca 


\begin{abstract}
Objective: The demand for effective psychological treatments for depression, anxiety, and heightened stress is far outstripping their supply. Accordingly, internet delivered, self-help interventions offer hope to many people, as they can be easily accessed and at a fraction of the price of face-to-face options. Mindfulness and self-compassion are particularly exciting approaches, as evidence suggests interventions that cultivate these skills are effective in reducing depression, anxiety, and heightened stress. We examined the efficacy of a newly developed program that combines mindfulness and self-compassion exercises into a brief self-guided intervention (Mind-OP). The secondary aim of this study was to investigate the feasibility of conducting a randomized-controlled trial entirely on a popular crowdsourcing platform, Amazon's Mechanical Turk (MTurk). Methods: We randomized 456 participants reporting heightened depression, anxiety, or stress to one of two conditions: the 4-week Mind-OP intervention $(n=227)$ or to an active control condition $(n=229)$ where participants watched nature videos superimposed onto relaxing meditation music for four consecutive weeks. We administered measures of anxiety, depression, perceived stress, dispositional and state mindfulness, self-compassion, and nonattachment. Results: Intent-to-treat and per-protocol analyses revealed that, compared to participants in the control condition, participants in the Mind-OP intervention condition reported significantly less anxiety and stress at the end of the trial, as well as significantly greater mindfulness, self-compassion, and nonattachment.
\end{abstract}

Conclusions: Mind-OP appears efficacious in reducing anxiety symptoms and perceived stress among MTurk participants. We highlight issues (e.g., attrition) related to feasibility of conducting randomized trials on crowdsourcing platforms such as MTurk. 
Keywords: mindfulness, self-compassion, internet interventions, crowdsourcing, MTurk,

depression, anxiety, stress, self-help 


\section{Examining the Efficacy of an Online Program to Cultivate Mindfulness and Self- Compassion Skills (Mind-OP): Randomized Controlled Trial on Amazon's Mechanical}

\section{Turk}

As many as $50 \%$ of people in developed nations report significant symptoms of depression or anxiety (Karsten et al., 2011; Kessler \& Bromet, 2013; Remes et al., 2016). Even when such symptoms are not meeting formal diagnostic thresholds, they are associated with significant impairment in day-to-day functioning, and high societal and personal costs (Cuijpers et al., 2007; 2013; Haller et al., 2014). Although perceived stress is not pathological in itself, chronically high stress has been robustly associated with psychological (Lee, 2012) and physiological disorder symptoms (Beshai et al., 2017). Although several efficacious treatments to manage depression, anxiety, and high perceived stress exist, the vast majority of people who would benefit from such treatments never seek them, as there are a number of barriers that prevent them from doing so (Mohr et al., 2010). For example, many patients report that financial and time constraints, lack of availability of services, and inflexibility of the treatment are major barriers to accessing treatments (Mohr et al., 2010). The Lancet Psychiatry Commission on Psychological treatments (Holmes et al., 2018) identified this lack of access to effective treatments as a major challenge.

Mindfulness - defined as paying purposeful attention to present-moment experiences with an attitude of openness, acceptance, and balance (Kabat-Zinn, 1982) - has garnered increasing scientific and public interest over the last two decades. Several mindfulness-based interventions have been designed to help those with elevated stress, depression, and anxiety (Chiesa \& Serretti, 2009; Crane et al., 2017), and many of such interventions appear efficacious for such conditions (Grossman et al., 2004; Strauss et al., 2014). In addition to mindfulness, self- 
compassion is another concept that has gained scientific and clinical attention of late (Neff, 2003a). Self-compassion is defined as being aware and moved by one's own suffering, a desire to respond with kindness toward one's own suffering and see it as part of the human condition (Neff, 2003b). Self-compassion and compassion-based interventions also appear efficacious for improving several mental health indices (Kirby et al., 2017).

To improve access to treatment, mental health professionals have begun to develop online interventions with the hope of mitigating several of these barriers to accessing quality care. Self-directed internet interventions are particularly attractive as they address many barriers to receiving mental health care (e.g., patient desire to manage problems independently, limited finances, time constraints, transportation or mobility challenges, poor access to providers, and concerns about privacy and stigma; Josephine et al., 2017). There is growing and strong evidence that self-directed internet interventions are effective in reducing symptoms of anxiety and depression. In fact, the effects of many of these interventions in reducing symptoms of anxiety and depression approximate those of traditional, face-to-face evidence-based psychotherapy, with effect sizes that are typically falling in the medium to large range (Beshai et al., 2016).

Given the need for improved access and dissemination of evidence-based psychological treatments, researchers have examined the efficacy of online forms of mindfulness and compassion-based interventions. There have been several of such trials to date. For example, Cavanagh et al. (2013) found that students assigned to their brief online mindfulness intervention reported significantly less anxiety depression symptoms, and perceived stress compared to those assigned to a waitlist control condition. Similarly, Krusche et al. (2013) found their online mindfulness intervention to be efficacious in lowering depression and anxiety symptoms as well as perceived stress. A recent meta-analysis confirmed the efficacy of online mindfulness 
interventions, with an overall small to moderate effect on depression and anxiety symptoms, and a large effect on stress (Spijkerman \& Bohlemeijer, 2016). Importantly, the researchers found only two trials (Pots et al., 2016; Trompetter et al. 2015) that used an active control condition, and several trials had small sample sizes.

Similarly, researchers have examined the efficacy of online delivery of compassion interventions. Smeets et al. (2014) found that those randomized to a brief online self-compassion intervention evidenced significant increases in self-compassion, mindfulness, optimism, and selfefficacy, and decreases in rumination compared to those randomized to an active control condition. In another trial, researchers found that participants receiving a self-guided online selfcompassion intervention reported significant increases in self-compassion and happiness, and significant decreases in perceived stress, depression, and anxiety (Finlay-Jones, et al., 2017).

\section{Overview}

Given the increasing need for scalable, easy-to-administer, and effective psychological treatments for anxiety, depression, and heightened perceived stress, we created a brief, nonproprietary 4-week online mindfulness-based program for mild to moderate levels of depression and anxiety symptoms, and moderate perceived stress. The program is called Mind-OP, and combines psychoeducational videos, meditative exercises designed to cultivate mindfulness and self-compassion, as well as motivational interviewing and decisional control exercises to increase engagement in the self-guided program. We anticipated participants randomized to the Mind-OP intervention would exhibit lower symptoms of anxiety and depression, and lower stress at the end of the assessment period than those randomized to the active control condition.

Importantly, we tested the efficacy of this program against a strong active control condition (watching and paying attention to nature videos; Mayer et al., 2009). Further, we 
examined these effects among a sample that is more representative of the general population than the commonly employed student samples (Berinsky et al., 2012; Chandler \& Shapiro, 2016). The secondary goal was to examine of feasibility of developing and validating online treatment modules using online participant pools such as Amazon Mechanical Turk. Although crowdsourcing platforms have been used for cross-sectional and descriptive clinical research, it is very rarely used for longitudinal research, and even more rarely used for randomized clinical trials.

\section{Method}

\section{Participants and Recruitment}

This trial corresponds to a pre-registered report (AsPredicted file \#18806; https://aspredicted.org/blind.php? $\mathrm{x}=\mathrm{b} 79 \mathrm{ky} 6$ ). We recruited participants through Amazon's Mechanical Turk's TurkPrime, an online crowdsourcing website (Litman et al., 2017). Crowdsourcing platforms have been used to date in several behavioral and clinical studies (e.g., Beshai, Mishra, Meadows, Parmar, \& Huang, 2017; Chandler \& Shapiro, 2016); however, by far the majority of clinical research conducted on such platforms is cross-sectional and descriptive in nature. Data collection for the trial was completed between April and September of 2019. All participants were compensated for their participation. To improve participant retention, compensation was backloaded, with the highest amount presented in the final week of assessment. Initially, and out of fears of duly influencing engagement with and outcomes of the intervention, we compensated participants in Waves 1 and 2 with $\$ 1.5 /$ week (total payment of $\$ 9$ USD); however, and given the high attrition rates, we increased payment to 2.5 per week (total payment of $\$ 15.00$ USD). The latter payment is commensurate with compensation rates in most crowdsourcing studies (Chandler \& Shapiro, 2016). Informed consent was obtained from all 
participants prior to data collection. This study was approved by the University of Regina's Ethics Research Board (File \#2018-158).

Several online mindfulness interventions evidenced medium effects when compared to inactive control conditions on main outcomes of interest (Spijkerman \& Bohlemeijer, 2016). Accordingly, and given the conservative nature of our design, sample size estimates were calculated using $\mathrm{G}^{*}$ Power (Faul et al., 2009), based on a small effect between groups across time $(\mathrm{f}=.10)$. The analysis indicated a total sample size of 164 participants to detect a small effect with power of .80 . We anticipated $50 \%$ eligibility and $50 \%$ attrition, and so, we planned to recruit a total of 700 participants (for an anticipated 175 protocol adherent participants).

\section{Eligibility and Randomization}

Eligibility requirements were that all participants reside in an English-speaking country (i.e., Canada, United States, United Kingdom, New Zealand, and Australia), be 18 years-of-age or older, and score eight or higher on the screening measures of depression or anxiety, and/or 14 or higher on the perceived stress scale (PSS). A total of 606 participants were initially recruited. Of these, 456 participants (female $n=200 ; 43.9 \%$ ) were eligible and provided consent to be part of the trial, and were subsequently randomized into the Mind-OP intervention condition $(n=$ $227 ; M_{\text {age }}=36.11 ; 46.3 \%$ female $)$ or active control condition $(n=229 ; M$ age $=34.16 ; 41.5 \%$ female). Table 1 provides a summary of pertinent sample demographics. Chi-square analyses revealed no significant differences in the distribution of gender, ethnicity, or education attainment across the two conditions ( $p=.56$ to .80$)$. A t-test revealed a significant difference in mean age between conditions, $(t(454)=1.98, p=.049)$.

\section{Primary Outcome Measures}


Generalized Anxiety Disorder - 7 (GAD-7; Spitzer et al., 2007) is a seven-item selfreport questionnaire that assesses the presence and severity of generalized anxiety symptoms over the past week. Respondents answer each of the seven items on a four-point Likert-type scale, from 0, or "Not at all", to 3, or "Nearly every day". Higher scores are indicative of greater distress. The GAD-7 appears to be reliable and valid among general population samples (Löwe et al., 2008), and is also sensitive to change (Beard \& Björgvinsson, 2014). Researchers have found cut-off scores of 5-10 on the GAD-7 to have optimal sensitivity and specificity in diagnosing generalized anxiety disorder (Kujanpää et al., 2014; Wild et al., 2014). In the current sample, the GAD-7 possessed a pre-treatment Cronbach's alpha of .89 .

The Patient Health Questionnaire - 9 (PHQ-9; Spitzer et al., 2000) is a nine-item selfreport measure that assesses depressive symptoms over the past two weeks. The PHQ was developed in accordance with criteria for Major Depressive Episode in the fourth edition of the DSM (DSM-IV). Participants responded to each of the nine items (e.g., "Feeling down, depressed, or hopeless") on a four-point Likert-type scale, ranging from 0 (Not at all) to 3 (Nearly everyday). Higher total scores were indicative of greater distress. The PHQ-9 has excellent reliability and validity among general population members (Kroenke et al., 2010), and appears sensitive to change (Löwe et al., 2004). Researchers found that cut-scores of 8-11 on the PHQ-9 have optimal sensitivity and specificity in diagnosing depression (Manea, Gilbody, \& McMillan, 2012). In the current sample, the PHQ-9 possessed a pre-treatment Cronbach's alpha of .88 .

The Perceived Stress Scale (PSS; Cohen, Kamarck, \& Mermelstein, 1983) is a 10-item self-report measure of perceived stress (PSS; Cohen, Kamarck, \& Mermelstein, 1983) recording how respondents appraised their lives over the previous month as stressful, unpredictable, 
uncontrollable, and overloaded. Higher scores on the PSS reflect increased levels of stress. The PSS is scored on a 5-point scale (from $0=$ 'never' to $4=$ 'very often') summed into a total score (range 0-40). Researchers have divided scores on the PSS into three categories of severity: mild stress (0 - 13), moderate stress (14-26), and high stress (26-40; Rasheed et al., 2017). This scale has demonstrated good reliability, validity, and sensitivity to change (Hewitt et al., 1992). In the current sample, the PSS had a pre-treatment Cronbach's alpha of .78.

\section{Secondary Outcome Measures}

The Five Facet Mindfulness Questionnaire - 15 (FFMQ-15; Gu et al., 2016) is a 15item self-report measure of dispositional mindfulness in five domains: Observing (ability to observe internal and external experiences), Describing (ability to label experiences), Acting with Awareness (ability to focus on experiences without acting automatically), Non Judging (ability to not evaluate internal and external experiences), and Non Reacting (ability to allow experiences to flow with equanimity). Participants responded to each of the 15 items on a 5-point Likert scale from 1, or Never or rarely true to 5, or Very often or always true, and after reversal of negatively keyed items, higher scores are indicative of greater dispositional mindfulness. The FFMQ-15 is adapted from the longer 39-item version (Baer et al., 2008), and both have excellent psychometric properties when used with general population participants and both are sensitive to change samples (Baer et al., 2008; Gu et al., 2016). In the current sample, the FFMQ-15 possessed a pre-treatment Cronbach's alpha of .76.

The Self-Compassion Scale - Short Form (SCS-SF; Raes et al., 2011) is a 12-item selfreport measure of dispositional self-compassion. The SCS-SF derived from the original version of SCS which includes 26 items (Neff, 2003). Each item was rated on a scale from 1 ("Almost never”) to 5 Almost always. Total scores are means of all the items, and after reversing 
negatively worded items, and higher scores indicate higher dispositional mindfulness. Previous studies have reported adequate psychometric properties of the SCS-SF and have shown that it has a high correlation with the long form of the scale (Raes et al., 2011). The SCS appears sensitive to change (Williams et al., 2014). In the current study, the SCS-SF demonstrated an adequate Cronbach's alpha of .87.

The Nonattachment Scale - Short Form (NAS-SF; Chio, Lai, \& Mak, 2018) is an eight-item short form of the original NAS (Sahdra, Shaver, \& Brown, 2010). The items assessed the Buddhist concept of dispositional nonattachment, or ability to not cling to impermanent mental or physical forms (Choi et al., 2018; Sahdra et al., 2010). Items (e.g., "I can accept the flow of events in my life without hanging onto them or pushing them away") are assessed on a 6point Likert-type scale from 1, or Disagree Strongly to 6 or Agree Strongly. Higher scores indicate higher nonattachment. The original scale and the short form appear to have strong reliability and validity (Choi et al., 2018; Sahdra et al., 2010). In the current sample, the NAS-SF possessed a pre-treatment Cronbach's alpha of .88 .

The Toronto Mindfulness Scale (TMS; Lau et al., 2006) is a 13-item measure assessing state mindfulness. The scale items assessed decentring (e.g., I experienced myself as separate from my changing thoughts and feelings) and curiosity ("I was more concerned with being open to my experiences than controlling or changing them") during meditative states. Each item is assessed on a 5-point Likert scale from 0 or Not at all" to 4 or "Very much". Total scores represent item response summation, and higher scores are indicative of greater state mindfulness. The scale has excellent reliability and validity (Lau et al., 2006) and is sensitive to change (Bieling et al., 2012). In the current sample, the TMS possessed a Cronbach's alpha of $\alpha=.93$. 


\section{Knowledge/Engagement Measures}

At the end of each week, participants in either condition responded to two multiple choice or true and false knowledge questions related to the content of each week (Moncher \& Prinz, 1991). For example, participants in the Mind-OP intervention condition were asked "Which of the following is NOT a quality of mindful attention?" and provided with four answer choices to which there is one correct response. Participants in the control condition were asked questions related to the words that were embedded in each of the nature videos (see description

of the active control condition below). For example, participants who completed the first week of the active control condition were asked "Which of the following words was shown on the side of the screen during the video?" and provided with four answer choices to which there was one correct response. In total, participants in either condition were asked eight knowledge questions throughout the duration of the study, and protocol fidelity was achieved when participants scored 5 or higher on such knowledge questions.

Finally, and to improve data quality, we administered two attention check questions at the end of the pre and post-assessment sessions. The questions promoted participants to indicate on a seven point Likert-type scale (where 1 was "Not at all attentive" and 7 was "Very attentive") how much attention and care they devoted in completing the measures (where 1 was "Not at all attentive" and 7 was "Very attentive"), and a yes/no format question asking whether their data should be retained. Attentiveness was defined as a score of 4 or higher on the first question and a "Yes" response on the second (Meade \& Craig, 2012).

\section{Adherence and Acceptability Measures}

At the beginning of modules 2, 3, 4, and of the post-assessment, participants were asked to indicate on a sliding visual scale from 0 to 100 how many minutes of meditation practice they 
engaged in. Secondly, at the end of each module, participants were asked to rate the quality of each module from 1 (poor) to 10 (excellent). Participants were also invited to provide a global rating of the program (1 to 10) at the post-assessment stage.

\section{Mind-OP Intervention}

Mind-OP is an entirely self-guided, brief online intervention that is hosted on Qualtrics. The intervention includes four modules/lessons, each focusing on a new aspect of mindfulness or self-compassion practice. The modules themselves are a combination of psychoeducational videos (designed to introduce a new concept), followed by audio-guided meditations that intend to cultivate the concept introduced in the psychoeducational videos. Embedded at the end of each module is a scheduler that prompts participants to schedule meditations throughout the week, as well as motivational interviewing inspired exercises to enhance decisional control and commitment to the weekly practice. For example, participants are asked to indicate how confident they are they will follow through with the scheduled practice, what might get in the way of their scheduled practice, and think briefly of potential solutions to or ways around identified obstacles to practicing. Each module is administered on a weekly basis, for a total of four weeks.

Module one (Week 1) is comprised of two videos (each approximately 5 minutes long) and one guided meditation (5 minutes). The first video contained general mental health psychoeducation related to anxiety, depression, and stress, while the second video introduces the concept of mindfulness. Participants are then guided to complete a guided mindfulness meditation of the breath. Module two (Week 2) then focuses on attention to body and thoughts. The first video ( $~ 5$ minutes) discusses how attention is limited, and how mindfulness can train the "attentional muscle" to make it more purposeful, balanced, and accepting. This 
psychoeducational video is followed by a guided audio of a body scan meditation ( $~ 6$ minutes $)$. This meditation is then followed by another psychoeducational video related to paying attention to the flow of thoughts ( $\sim 5$ minutes), again, followed by an audio guided meditation focused on mindfulness of thoughts.

Module three (Week 3) is also comprised of two psychoeducation videos and two guided meditations (each approximately 5-6 minutes long). The first psychoeducational video introduces the concept of self-compassion, specifically the mindfulness subcomponent of self-compassion (Neff, 2003). This psychoeducational video is followed by a guided meditation that focuses on sitting with uncomfortable emotions with equanimity, balance, and acceptance. Since this meditation may be associated with increased negative emotions, participants are reminded to return to the breath if they feel overwhelmed. Further, participants are guided to engage in loving kindness at the end of the meditation, to counteract the potential uncomfortable nature of negative emotions to which they may have been exposed during the meditation. The second psychoeducation video introduces the self-compassion component of common humanity, which is then followed by a guided meditation that guides participants to extend loving kindness toward imagined others.

Module four (Week 4) focuses entirely on the self-kindness component of selfcompassion (Neff, 2003). The module is comprised of one psychoeducational video ( $\sim 6$ minutes) introducing said concept and differentiating it from self-pity. After watching the video, participants are invited to answer questions that help differentiate self-kindness and its effects from self-pity. In the final psychoeducational video, participants are invited to practice selfkindness in relation to a specific stressful event or situation. This video prompts participants to complete an adapted Self-Compassion Break exercise, as inspired by Neff and other colleagues' 
work in the area (Finlay-Jones et al., 2017; Kirby, 2017; Neff \& Germer, 2013). The final guided meditation invites participants to complete a loving-kindness meditation directed entirely toward self at different developmental timepoints (e.g., "imagine yourself as a 5-year old child").

\section{Active Control Condition}

Participants randomized to the active control condition were guided to watch one video per week, for the 4-week duration of the intervention. Each video featured 40 high quality, peaceful stock nature images that were presented in a slideshow (with each image appearing on screen for 6 seconds). Each video was approximately four minutes long and featured the same guided audio music soundtrack as the Mind-OP guided meditations. Finally, participants were presented with two words during each video that were small, white font, in the corner of the screen, and appeared for only a few seconds at random intervals throughout.

\section{Procedure}

All trial tasks were distributed online through TurkPrime (Litman, Robinson, \& Abberbock, 2017), an MTurk-based crowdsourcing platform. Study surveys and weekly modules were hosted on Qualtrics. After providing consent, participants completed the pre-intervention measures, including a demographic information form, the PHQ-9, GAD-7, PSS, FFMQ-15, SCSSF, NAS-SF, and TMS.

In the week between completion of the pre-intervention survey and distribution of Module 1/Week 1 tasks, trained research assistants computed scores on the PHQ-9, GAD-7, and PSS. Those eligible (PHQ-9 or GAD-7 of 8 or higher or PSS of 14 and higher), were randomized into either the Mind-OP or Control condition using SPSS's 1:1 ratio Random Case Selection procedure (Afrin, 2012). Ineligible participants were contacted via TurkPrime, thanked, and provided with links to the four modules to use at their own discretion. Eligible participants were 
contacted and provided with Week 1 materials. Trial tasks were distributed every Friday throughout the 5-week duration of the trial, with one email reminder sent out each Wednesday. At the end of the trial, all participants in the control condition were provided links to the MindOP intervention modules.

\section{Statistical Analysis Plan}

Data management and preliminary checks. To examine the normality of the dependent variables, we explored the skewness and kurtosis for all four measures both pre and post intervention (Tabachnick \& Fidell, 2007). Further, using a series of chi-square and t-test analyses, we examined demographic and psychological variable differences between completers and those who were randomized but never completed or adhered to the protocol .

Main Outcome Analyses. We conducted two sets of main outcome analyses, one in accordance with intention-to-treat (ITT) methodology involving all randomized participants, and one in accordance with per-protocol methodology. For the per-protocol analyses, only those who completed all modules and demonstrated high treatment fidelity to the Mind-OP intervention $(n$ $=68)$ or control $(n=91)$ were analyzed.

ITT analyses were conducted in accordance with Mixed Linear Modeling (MLM), where we examined main effects of time (six week of assessment; pre-intervention, weeks 1, 2, 3, 4, and post-assessment) and group allocation (intervention vs. control) on main outcome measures (GAD-7, PHQ-9; PSS). Further, and to assess main outcome hypotheses, we assessed the interaction effect of time with group allocation. A significant interaction indicates significant differences in the slope of change between individuals randomized to the intervention and control conditions. Further, all analyses accounted for random effects of intercept (individual differences in starting point), as well as utilized unstructured covariances. Additions of intercept 
and unstructured covariance significantly improved model fit in all three main outcome MLM analyses in accordance with a Chi-square significance test (Groeneveld \& Kovac, 1990; McLean et al., 1991).

Per-protocol analyses were conducted using a mixed factor repeated-measures analysis of variance (ANOVA), wherein there were one between-subject (randomization group) and one within-subject (pre-post assessment) main effect, and one interaction effect. Significant interaction effects were followed up by a series of paired samples t-tests to examine effect sizes related to change from pre- to post-assessment within each of the randomization conditions. Secondary Outcome Analyses. A series of repeated-measures ANOVAs were conducted to examine differences across time and condition on measures of dispositional mindfulness (FFMQ-15), self-compassion (SCS-SF), nonattachment (NAS-SF), and state-level mindfulness during a recent meditation session (TMS). We followed up significant interaction effects with a series of paired-samples t-tests to examine effect size related to the change over time within each condition. We also conducted several exploratory paired samples t-tests to examine withinsubject effect size differences on the facets of mindfulness (FFMQ) and the decentring and curiosity subfactors of the TMS within each randomization condition.

We also conducted exploratory descriptive analyses of between module meditation time, and module and program ratings. Alpha/significance level for all analyses was set at .05 and were conducted using SPSS v. 25.

\section{Results}

\section{Normality Checks}

Skewness for all measures (pre and post intervention) ranged from -.55 to .62, all within the suggested range of +/- 1 for normal distributions (Tabachnick \& Fidell, 2007). Similarly, all 
kurtosis statistics for the dependent measures were within the range expected of a normal distribution, ranging from -.72 to .76 .

\section{Attrition and Adherence}

Figure 1 depicts participant flow throughout all timepoints in the study. Attrition rates were high across both conditions, but were higher for the treatment condition, $\chi_{2}(1)=4.80, p=$ .028. Analyses revealed no significant differences between those who adhered to the protocol compared to those who did not in age, $t(454)=-.82, p=.41$, gender, $\chi^{2}(2)=0.28, p=.87$, ethnicity, $\chi_{2}(10)=9.52, p=.48$, previous meditation experience, $\chi_{2}(1)=0.48, p=.49$, and previous mental health condition, $\chi_{2}(1)=2.51, p=.11$. There was a significant difference between those adhering to the protocol compared to those not adhering in distribution of highest levels of educational attainment, $\chi_{2}(5)=12.63, p=.027$.

A series of independent samples t-test analyses revealed no significant differences between those who adhered to the protocol compared to those who did not adhere on pretreatment levels of anxiety, $t(454)=1.49, p=.14$, depression, $t(454)=1.49, p=.14$, stress, $t(454)=-0.68, p=.52$, or dispositional mindfulness, $t(454)=1.23, p=.14$.

There were significant differences between those who adhered versus those who did not on measures of self-compassion, $t(454)=2.79, p=.005$, nonattachment, $t(454)=2.68, p=.007$, and state mindfulness, $t(454)=3.26, p<.001$. These analyses revealed that those who adhered to the treatment scored significantly lower than those who did not adhere to the treatment on the SCS-SF, NAS-SF, and TMS. Descriptive statistics of primary and secondary outcome measures, stratified by condition among protocol adherent participants, are summarized in Table 2.

\section{Intent-To-Treat Primary Outcomes}


Anxiety. MLM analyses results are summarized in Table 3. Analyses revealed significant effects of Time (week of assessment) and Group allocation on GAD-7 scores. The analysis also revealed a significant Time by Group interaction, indicating a significant difference in slope of change between those allocated to the treatment and control conditions over time. Allocation to the Mind-OP treatment condition was associated with a decreased of 1.78 points $(p=.043)$ on the GAD-7 across time and compared with the control condition (See Figure 2).

Depression. MLM analyses revealed a significant effect of Time (week of assessment) on PHQ-9 scores. There was no significant effect of group membership on PHQ-9 scores. There was a significant Time by Group interaction, indicating a significant difference in slope of change between those allocated to the treatment and control conditions (See Figure 3).

Stress. MLM analyses revealed significant effects of Time (week of assessment) and group allocation on PSS scores. Analyses also revealed a significant Time by Group interaction, indicating a significant difference in slope of change between those allocated in the treatment and control conditions. Allocation to the Mind-OP treatment condition was associated with a decrease of -3.65 points $(p<.001)$ on the PSS across time and compared to the control condition (See Figure 4).

\section{Per-Protocol Primary Outcomes}

A Repeated-measures ANOVA revealed a significant main effect of time, $F(1,157)=$ $60.93, p<.001, \eta_{\mathrm{p} 2}=.28$, and group membership, $F(1,157)=6.34, p=.01, \eta_{\mathrm{p} 2}=.04$, on GAD-7 scores. There was a significant time by group interaction on GAD-7 scores, $F(1,157)=4.75, p=$ $.031, \eta_{\mathrm{p} 2}=.03$

There was a significant main effect of time on PHQ-9 scores, $F(1,157)=70.73, p<.001$, $\eta_{\mathrm{p} 2}=.31$; however, the main effect of group membership on PHQ-9 scores was not significant, 
$F(1,157)=2.57, p=.11, \eta_{\mathrm{p} 2}=.02$. There was no significant time by group allocation effect on PHQ-9 scores, $F(1,157)=1.76, p<.001, \eta_{\mathrm{p} 2}=.01$.

Analyses revealed significant main effects of time, $F(1,157)=152.75, p<.001, \eta_{\mathrm{p} 2}=$ .49 , and group membership, $F(1,157)=7.25, p=.008, \eta_{\mathrm{p} 2}=.044$, on PSS scores. The analyses also revealed a significant time by group interaction effect on PSS scores, $F(1,157)=15.17, p<$ $.001, \eta_{\mathrm{p} 2}=.09$

\section{Per-Protocol Secondary Outcomes}

A repeated-measures ANOVA revealed a significant main effect of time, , $F(1,157)=$ $59.44, p<.001, \eta_{\mathrm{p} 2}=.28$ on FFMQ-15 scores. There was no significant main effect of group membership on FFMQ-15 scores, $F(1,157)=0.39, p=.53, \eta_{\mathrm{p} 2}=.003$. The analysis revealed a significant time by group effect on FFMQ-15 scores, $F(1,157)=5.09, p=.025, \eta_{\mathrm{p} 2}=.03$.

Analyses revealed a significant main effect of time on SCS-SF scores, $F(1,157)=65.51$, $p<.001, \eta_{\mathrm{p} 2}=.29 ;$ however, there was no significant main effect of group membership on SCSSF scores, $F(1,157)=1.96, p=.16, \eta_{\mathrm{p} 2}=.01$. There was a significant time by group interaction on the SCS-SF, $F(1,157)=7.67, p=.006, \eta_{\mathrm{p} 2}=.05$.

Analyses revealed a significant time effect on NAS scores, $F(1,157)=69.70, p<.001$, $\eta_{\mathrm{p} 2}=.31$. There was no main effect of group membership on NAS scores, $F(1,157)=0.20, p=$ $.65, \eta_{\mathrm{p} 2}=.001$. The analysis revealed a significant time by group interaction on the NAS, $F(1$, 157) $=3.99, p=.047, \eta_{\mathrm{p} 2}=.025$.

The final repeated measures analysis revealed a significant main effect of time on TMS scores, $F(1,157)=13.22, p<.001, \eta_{\mathrm{p} 2}=.078$. There was no main effect of group membership on TMS scores, $F(1,157)=1.92, p=.17, \eta_{\mathrm{p} 2}=.012$. There was a significant time by group interaction effect on TMS scores, $F(1,157)=11.61, p<.001, \eta_{\mathrm{p} 2}=.069$. 


\section{Exploratory Paired Samples Tests}

Exploratory paired samples t-test analyses indicated that participants allocated to the intervention condition experienced significant changes from pre to post with effect sizes that ranged from $d=.57$ (TMS) to 1.45 (PSS). By comparison, participants in the active control condition experienced changes with effect sizes ranging from $d=.02$ (TMS) to .63 (PSS). Similarly, effect sizes of change from pre to post on FFMQ-15 and TMS subscales for people in the intervention condition ranged from .30 (FFMQ-Describe) to .68 (TMS-Decentring). These results are summarized in Tables 4 and 5.

\section{Between Module Meditation and Program Acceptability}

Mean meditation practice times between modules 1 to 2,2 to 3,3 to 4 , and 4 to postassessment were $20.66(\mathrm{SD}=15.90), 23.91(\mathrm{SD}=18.71), 23.21(\mathrm{SD}=18.5)$, and 27.95 minutes $(\mathrm{SD}=30.60)$, respectively

Module ratings were $7.91(\mathrm{SD} .1 .75), 8.03(\mathrm{SD}=1.73), 8.05(\mathrm{SD}=1.68)$, and $8.24(\mathrm{SD}=$ 1.59) for modules 1 to 4 , respectively. Overall program rating was 8.23 ( $\mathrm{SD}=1.49$ ).

\section{Discussion}

In this randomized trial, we examined the efficacy of an online program designed to cultivate mindfulness and self-compassion skills, and to reduce symptoms of anxiety, depression, and heightened subjective stress. The intervention itself was highly novel, combining both mindfulness and self-compassion approaches and exercises. Further, this is one of the first trials of its kind to be conducted entirely on a crowdsourcing platform such as Amazon's Mechanical Turk. Accordingly, this was a proof-of-concept, feasibility study. The intervention itself had encouraging results and had medium-to-large effects on reducing anxiety symptoms and managing stress, as well as on the secondary outcomes of dispositional and state mindfulness, 
self-compassion, and non-attachment. Further, we tested the effects of the intervention against a relatively stringent active control condition, wherein participants watched nature videos, superimposed onto the meditation music from our Mind-OP meditation videos (Mayer et al., 2009).

Consistent with other trials of self-guided interventions (Beshai et al., 2016), attrition was high in both conditions, but was higher among those randomized to Mind-OP. Complete adherence rates (i.e., completed all modules, while paying attention and engaging with the material) in the Mind-OP condition were 30\%, which is consistent with other studies which found adherence of 14-50\% in self-guided interventions for depression (Cuijpers et al., 2011; Karyotaki et al., 2015). It is noteworthy that the differences in attrition among those randomized to the intervention and control conditions can be accounted for by the higher attrition rates between baseline and initiation of Module 1. If the increased attrition at this specific time point is accounted for, attrition rates were similar across conditions at $50 \%$ (i.e., $50 \%$ of those who initiated week 1 of either condition had complete adherence).

Also, consistent with other trials of online mindfulness and self-compassion interventions, we found that Mind-OP had the largest effects on perceived stress (Spijkerman et al., 2016). The treatment evidenced small effects for anxiety symptoms across time and compared to the active control condition, but did not appear to be effective for depression symptoms when compared with the active control procedure. There is evidence to suggest that therapist-guided interventions are more effective for the treatment of depression than are entirely self-guided interventions (Gellatly et al., 2007). Further, per-protocol analyses demonstrated that participants randomized to Mind-OP showed significant increases in secondary outcomes of dispositional mindfulness, self-compassion, non-attachment, and state mindfulness compared to controls. 
These are also consistent with results of other trials which showed that online mindfulness and self-compassion interventions are efficacious in raising these important secondary and process outcomes (Smeets et al., 2014; Trompetter, et al., 2016).

The current trial had several strengths. First, the program itself was unique in combining evidence-based psychoeducational videos and exercises from disparate fields (mindfulness, selfcompassion, decisional control, and goal-setting theories). Secondly, there are currently very few published works that compared online mindfulness or compassion-based interventions with an active control condition, with by far the majority of existing trials having compared treatments with passive waitlist conditions. The results of the trial clearly demonstrate that even paying attention to a brief nature video and completing study measures on a weekly basis evidenced small-moderate effects on both primary and secondary outcomes. Moreover, and to the best of our knowledge, this is the first study of its kind to be completed entirely on a crowdsourcing platform such as MTurk. Despite the high attrition rates, this trial was proof-of-concept that such recruitment methods are indeed feasible and have immense potential in clinical research (Chandler \& Shapiro, 2016). Also, despite high attrition rates, we still had relatively large sample sizes of participants adhering to the trial protocol $(n=159)$, which lends more confidence in the obtained results.

The study also suffers from several limitations that pave the way for future trials. First, we did not compare the results of the intervention and active control conditions to those of a waitlist/passive control. As such, it was not possible to ascertain the effects of these conditions compared to receiving no treatment at all. Second, a major limitation is we only assessed participants post-intervention, and thus, we did not have a longer follow-up to evaluate whether the results hold across time (Spijkerman et al., 2016). Third, and as mentioned, attrition rates 
were demonstrably high. It is difficult to ascertain whether these high attrition rates were due to the non-acceptable nature of the intervention itself, or whether these rates were due to the nature of the recruitment platform, which is essentially a work-for-pay environment. It is noteworthy that some participants complained about the incentive structure, many of whom felt that a) the payment was too low, or b) that homework adherence (in the form of completing meditations) needs to be further incentivized through payment that is independent from payment for completing each module. Fourth, the wide net recruitment strategy using MTurk for a mental health intervention raises a few ethical concerns; given jurisdictional restrictions and lack of adequate resources, we could not closely monitor outcomes on an individual basis, nor could we respond to any crises that arose as result of the intervention or otherwise. With that said, this treatment was entirely self-guided, and so participants were free to engage or disengage from the treatment at their own discretion. Further, the treatment was pilot tested among a small sample of university students, and its safety was ascertained prior to deployment on MTurk. Accordingly, the potential mental health benefits of offering this intervention widely through MTurk far outweighed the potential risks, given the ubiquity of mental health concerns among this sample (Arditte et al., 2016).

Despite the enormous progress we have made in designing effective interventions for common mental health concerns, access to and engagement with these treatments has remained dismally low. Online, brief self-guided interventions hold great promise, given their scalability and their ability to address several of the barriers to access experienced by those who need these treatments most. Mindfulness and self-compassion are particularly promising, given their wide general appeal as well as the growing scientific evidence that backs their efficacy. Our treatment combines several evidence-based mindfulness and self-compassion techniques and packages 
them with other behavioral techniques for improved mental health and engagement. This treatment evidenced moderate effects for stress and small to moderate effects for anxiety when compared with an active control condition. Further, and as an added bonus, we were able to demonstrate that a randomized trial of this nature is feasible on a crowdsourcing recruitment platform such as MTurk. As such, we hope this trial paves the way for future clinical work using this versatile platform. 


\section{References}

Al Rasheed, F., Naqvi, A. A., Ahmad, R., \& Ahmad, N. (2017). Academic stress and prevalence of stress-related self-medication among undergraduate female students of health and nonhealth cluster colleges of a public sector university in Dammam, Saudi Arabia. Journal of pharmacy \& bioallied sciences, 9(4), 251. https://doi.org/10.4103/jpbs.JPBS_189_17

Arditte, K. A., Çek, D., Shaw, A. M., \& Timpano, K. R. (2016). The importance of assessing clinical phenomena in Mechanical Turk research. Psychological assessment, 28(6), 684. https://doi.org/10.1037/pas0000217

Baer, R. A., Smith, G. T., Lykins, E., Button, D., Krietemeyer, J., Sauer, S., ... \& Williams, J. M. G. (2008). Construct validity of the five facet mindfulness questionnaire in meditating and nonmeditating samples. Assessment, 15(3), 329-342. https://doi.org/10.1177/1073191107313003

Beard, C., \& Björgvinsson, T. (2014). Beyond generalized anxiety disorder: psychometric properties of the GAD-7 in a heterogeneous psychiatric sample. Journal of Anxiety Disorders, 28(6), 547-552. https://doi.org/10.1016/j.janxdis.2014.06.002

Berinsky, A. J., Huber, G. A., \& Lenz, G. S. (2012). Evaluating online labor markets for experimental research: Amazon. com's Mechanical Turk. Political Analysis, 20(3), 351368. https://doi.org/10.1093/pan/mpr057

Beshai, S., Mishra, S., Meadows, T. J., Parmar, P., \& Huang, V. (2017). Minding the gap: Subjective relative deprivation and depressive symptoms. Social Science \& Medicine, 173, 18-25. https://doi.org/0.1016/j.socscimed.2016.11.021 
Beshai, S., Wallace, L. M., Mcdougall, K. H., Waldmann, K., \& Stea, J. N. (2016). Reduced contact cognitive-behavioral interventions for adult depression: a review. The Journal of psychology, 150(2), 252-279. https://doi.org/10.1080/00223980.2015.1087376

Cavanagh, K., Strauss, C., Cicconi, F., Griffiths, N., Wyper, A., \& Jones, F. (2013). A randomised controlled trial of a brief online mindfulness-based intervention. Behaviour research and therapy, 51(9), 573-578. https://doi.org/10.1016/j.brat.2013.06.003

Chandler, J., \& Shapiro, D. (2016). Conducting clinical research using crowdsourced convenience samples. Annual Review of Clinical Psychology, 12, 51-82. https://doi.org/10.1146/annurev-clinpsy-021815-093623

Chio, F. H., Lai, M. H., \& Mak, W. W. (2018). Development of the nonattachment scale-short form (NAS-SF) using item response theory. Mindfulness, 9(4), 1299-1308. https://doi.org/10.1007/s12671-017-0874-z

Crane, R. S., Brewer, J., Feldman, C., Kabat-Zinn, J., Santorelli, S., Williams, J. M. G., \& Kuyken, W. (2017). What defines mindfulness-based programs? The warp and the weft. Psychological medicine, 47(6), 990-999. https://doi.org/10.1017/S0033291716003317

Cuijpers, P., Donker, T., Johansson, R., Mohr, D. C., van Straten, A., \& Andersson, G. (2011). Self-guided psychological treatment for depressive symptoms: a meta-analysis. PloS one, 6(6), e21274. https://doi.org/10.1371/journal.pone.0021274

Cuijpers, P., Smit, F., Oostenbrink, J., De Graaf, R., Ten Have, M., \& Beekman, A. (2007). Economic costs of minor depression: a population-based study. Acta Psychiatrica Scandinavica, 115(3), 229-236. https://doi.org/10.1111/j.1600-0447.2006.00851.x 
Cuijpers, P., Vogelzangs, N., Twisk, J., Kleiboer, A., Li, J., \& Penninx, B. W. (2013). Differential mortality rates in major and subthreshold depression: meta-analysis of studies that measured both. The British Journal of Psychiatry, 202(1), 22-27. https://doi.org/10.1192/bjp.bp.112.112169

Faul, F., Erdfelder, E., Buchner, A., \& Lang, A. G. (2009). Statistical power analyses using G* Power 3.1: Tests for correlation and regression analyses. Behavior Research Methods, 41(4), 1149-1160.

Finlay-Jones, A., Kane, R., \& Rees, C. (2017). Self-compassion online: A pilot study of an internet-based self-compassion cultivation program for psychology trainees. Journal of Clinical Psychology, 73(7), 797-816. https://doi.org/10.1002/jclp.22375

Friedrich, M. J. (2017). Depression Is the Leading Cause of Disability Around the World. JAMA, 317(15), 1517-1517. https://doi.org/10.1001/jama.2017.3826

Goodman, J. H. (2009). Women's attitudes, preferences, and perceived barriers to treatment for perinatal depression. Birth, 36(1), 60-69. https://doi.org/10.1111/j.1523536X.2008.00296.x

Groeneveld, E., \& Kovac, M. (1990). A generalized computing procedure for setting up and solving mixed linear models. Journal of Dairy Science, 73(2), 513-531. https://doi.org/10.3168/jds.S0022-0302(90)78699-7

Gu, J., Strauss, C., Crane, C., Barnhofer, T., Karl, A., Cavanagh, K., \& Kuyken, W. (2016). Examining the factor structure of the 39-item and 15-item versions of the Five Facet Mindfulness Questionnaire before and after mindfulness-based cognitive therapy for people with recurrent depression. Psychological Assessment, 28(7), 791. https://doi.org/10.1037/pas0000263 
Haller, H., Cramer, H., Lauche, R., Gass, F., \& Dobos, G. J. (2014). The prevalence and burden of subthreshold generalized anxiety disorder: a systematic review. $B M C$ psychiatry, 14(1), 128. https://doi.org/10.1186/1471-244X-14-128

Karsten, J., Nolen, W. A., Penninx, B. W., \& Hartman, C. A. (2011). Subthreshold anxiety better defined by symptom self-report than by diagnostic interview. Journal of Affective Disorders, 129(1-3), 236-243. https://doi.org/10.1016/j.jad.2010.09.006

Karyotaki, E., Kleiboer, A., Smit, F., Turner, D. T., Pastor, A. M., Andersson, G., ... \& Christensen, H. (2015). Predictors of treatment dropout in self-guided web-based interventions for depression: an 'individual patient data'meta-analysis. Psychological medicine, 45(13), 2717-2726. https://doi.org/10.1017/S0033291715000665

Kirby, J. N. (2017). Compassion interventions: The programmes, the evidence, and implications for research and practice. Psychology and Psychotherapy: Theory, Research and Practice, 90(3), 432-455.

Kirby, J. N., Tellegen, C. L., \& Steindl, S. R. (2017). A meta-analysis of compassion-based interventions: Current state of knowledge and future directions. Behavior Therapy, 48(6), 778-792. https://doi.org/10.1016/j.beth.2017.06.003

Krusche, A., Cyhlarova, E., \& Williams, J. M. G. (2013). Mindfulness online: an evaluation of the feasibility of a web-based mindfulness course for stress, anxiety and depression. $B M J$ open, 3(11), e003498. https://doi.org/10.1136/bmjopen-2013-003498

Kroenke, K., Spitzer, R. L., Williams, J. B., \& Löwe, B. (2010). The patient health questionnaire somatic, anxiety, and depressive symptom scales: a systematic review. General hospital psychiatry, 32(4), 345-359. https://doi.org/10.1016/j.genhosppsych.2010.03.006 
Kujanpää, T., Ylisaukko-Oja, T., Jokelainen, J., Hirsikangas, S., Kanste, O., Kyngäs, H., \& Timonen, M. (2014). Prevalence of anxiety disorders among Finnish primary care high utilizers and validation of Finnish translation of GAD-7 and GAD-2 screening tools. Scandinavian journal of primary health care, 32(2), 78-83. https://doi.org/10.3109/02813432.2014.920597

Lau, M. A., Bishop, S. R., Segal, Z. V., Buis, T., Anderson, N. D., Carlson, L., et al. (2006). The Toronto Mindfulness Scale: development and validation. Journal of Clinical Psychology, 62, 1445-1467. https://doi.org/10.1002/jclp.20326

Litman, L., Robinson, J., \& Abberbock, T. (2017). TurkPrime. com: A versatile crowdsourcing data acquisition platform for the behavioral sciences. Behavior research methods, 49(2), 433-442. https://doi.org/10.3758/s13428-016-0727-z

Löwe, B., Decker, O., Müller, S., Brähler, E., Schellberg, D., Herzog, W., \& Herzberg, P. Y. (2008). Validation and standardization of the Generalized Anxiety Disorder Screener (GAD-7) in the general population. Medical care, 46(3), 266-274. https://doi.org/10.1097/MLR.0b013e318160d093

Löwe, B., Unützer, J., Callahan, C. M., Perkins, A. J., \& Kroenke, K. (2004). Monitoring depression treatment outcomes with the patient health questionnaire-9. Medical care, 1194-1201. https://doi.org/10.1097/00005650-200412000-00006

Manea, L., Gilbody, S., \& McMillan, D. (2012). Optimal cut-off score for diagnosing depression with the Patient Health Questionnaire (PHQ-9): a meta-analysis. Cmaj, 184(3), E191E196. https://doi.org/10.1503/cmaj.110829 
Mayer, F. S., Frantz, C. M., Bruehlman-Senecal, E., \& Dolliver, K. (2009). Why is nature beneficial? The role of connectedness to nature. Environment and behavior, 41(5), 607643. https://doi.org/10.1177/0013916508319745

McLean, R. A., Sanders, W. L., \& Stroup, W. W. (1991). A unified approach to mixed linear models. The American Statistician, 45(1), 54-64. https://doi.org/10.1080/00031305.1991.10475767

Mohr, D. C., Hart, S. L., Howard, I., Julian, L., Vella, L., Catledge, C., \& Feldman, M. D. (2006). Barriers to psychotherapy among depressed and nondepressed primary care patients. Annals of Behavioral Medicine, 32(3), 254-258. https://doi.org/10.1207/s15324796abm3203_12

Moncher, F. J., \& Prinz, R. J. (1991). Treatment fidelity in outcome studies. Clinical Psychology Review, 11(3), 247-266.

Neff, K. (2003). Self-compassion: An alternative conceptualization of a healthy attitude toward oneself. Self and identity, 2(2), 85-101.

Neff, K. D., \& Germer, C. K. (2013). A pilot study and randomized controlled trial of the mindful self-compassion program. Journal of Clinical Psychology, 69(1), 28-44. https://doi.org/10.1002/jclp.21923

Remes, O., Brayne, C., Van Der Linde, R., \& Lafortune, L. (2016). A systematic review of reviews on the prevalence of anxiety disorders in adult populations. Brain and Behavior, 6(7), e00497. https://doi.org/10.1002/brb3.497

Smeets, E., Neff, K., Alberts, H., \& Peters, M. (2014). Meeting suffering with kindness: Effects of a brief self-compassion intervention for female college students. Journal of Clinical Psychology, 70(9), 794-807. https://doi.org/10.1002/jclp.22076 
Spijkerman, M. P. J., Pots, W. T. M., \& Bohlmeijer, E. T. (2016). Effectiveness of online mindfulness-based interventions in improving mental health: A review and meta-analysis of randomised controlled trials. Clinical Psychology Review, 45, 102-114. https://doi.org/10.1016/j.cpr.2016.03.009

Spitzer, R. L., Kroenke, K., Williams, J. B., \& Löwe, B. (2006). A brief measure for assessing generalized anxiety disorder: the GAD-7. Archives of Internal Medicine, 166(10), 10921097. https://doi.org/10.1001/archinte.166.10.1092

Spitzer, R. L., Williams, J. B., Kroenke, K., Hornyak, R., McMurray, J., \& Patient Health Questionnaire Obstetrics-Gynecology Study Group. (2000). Validity and utility of the PRIME-MD patient health questionnaire in assessment of 3000 obstetric-gynecologic patients: the PRIME-MD Patient Health Questionnaire Obstetrics-Gynecology Study. American Journal of Obstetrics and Gynecology, 183(3), 759-769. https://doi.org/10.1067/mob.2000.106580

Strauss, C., Cavanagh, K., Oliver, A., \& Pettman, D. (2014). Mindfulness-based interventions for people diagnosed with a current episode of an anxiety or depressive disorder: a metaanalysis of randomised controlled trials. PLoS One, 9(4), e96110. https://doi.org/10.1371/journal.pone.0096110

Trompetter, H. R., Bohlmeijer, E. T., Veehof, M. M., \& Schreurs, K. M. (2015). Internet-based guided self-help intervention for chronic pain based on Acceptance and Commitment Therapy: a randomized controlled trial. Journal of Behavioral Medicine, 38(1), 66-80. https://doi.org/10.1007/s10865-014-9579-0

Wild, B., Eckl, A., Herzog, W., Niehoff, D., Lechner, S., Maatouk, I., ... \& Löwe, B. (2014). Assessing generalized anxiety disorder in elderly people using the GAD-7 and GAD-2 
scales: results of a validation study. The American Journal of Geriatric

Psychiatry, 22(10), 1029-1038. https://doi.org/10.1016/j.jagp.2013.01.076

Williams, M. J., Dalgleish, T., Karl, A., \& Kuyken, W. (2014). Examining the factor structures of the five facet mindfulness questionnaire and the self-compassion scale. Psychological Assessment, 26(2), 407. https://doi.org/10.1037/a0035566 
Table 1

Descriptive Statistics of Trial Participants Stratified by Randomization Condition

\begin{tabular}{|c|c|c|c|}
\hline & $\begin{array}{l}\text { Total Sample } \\
(n=456)\end{array}$ & $\begin{array}{l}\text { Mind-OP Intervention } \\
(n=227)\end{array}$ & $\begin{array}{l}\text { Active Control } \\
(n=229)\end{array}$ \\
\hline Age & $M=35.13(S D=10.57)$ & $M=36.11(S D=11.16)$ & $M=34.16(S D=9.88)$ \\
\hline Gender & $\begin{array}{l}\text { Female } n=200 \\
(43.9 \%) \\
\text { Non-binary } n=2 \\
(0.4 \%)\end{array}$ & $\begin{array}{l}\text { Female } n=105 \\
(46.3 \%) \\
\text { Non-binary } n=1 \\
(0.4 \%)\end{array}$ & $\begin{array}{l}\text { Female } n=95(41.5 \%) \\
\text { Non-binary } n=1 \\
(0.4 \%)\end{array}$ \\
\hline \multicolumn{4}{|l|}{ Ethnicity } \\
\hline Western European & $n=192(42.1 \%)$ & $n=100(44.1 \%)$ & $n=92(40.2 \%)$ \\
\hline $\begin{array}{l}\text { Eastern European } \\
\text { Chinese } \\
\text { Latin American }\end{array}$ & $\begin{array}{l}n=104(22.8 \%) \\
n=14(3.1 \%) \\
n=41(9.0 \%)\end{array}$ & $\begin{array}{l}n=53(23.3 \%) \\
n=7(3.1 \%) \\
n=16(7.0 \%)\end{array}$ & $\begin{array}{l}n=51(10.9 \%) \\
n=7(3.1 \%) \\
n=25(10.9 \%)\end{array}$ \\
\hline Other & $n=105(22.6 \%)$ & $n=51$ & $n=54$ \\
\hline \multicolumn{4}{|l|}{ Country of Residence } \\
\hline $\begin{array}{l}\text { United States } \\
\text { Other }\end{array}$ & $\begin{array}{l}n=441(96.7 \%) \\
n=15(3.3 \%)\end{array}$ & $\begin{array}{l}n=221(97.4 \%) \\
n=6(2.6 \%)\end{array}$ & $\begin{array}{l}n=220(96.1 \%) \\
n=9(3.9 \%)\end{array}$ \\
\hline \multicolumn{4}{|l|}{ Education } \\
\hline $\begin{array}{l}\text { High School } \\
\text { Community } \\
\text { College }\end{array}$ & $\begin{array}{l}n=92(20.2 \%) \\
n=76(16.7 \%)\end{array}$ & $\begin{array}{l}n=52(22.9 \%) \\
n=39(17.2 \%)\end{array}$ & $\begin{array}{l}n=40(17.5 \%) \\
n=37(16.2 \%)\end{array}$ \\
\hline University & $n=224(49.1 \%)$ & $n=105(46.3 \%)$ & $n=119(52.0 \%)$ \\
\hline Master or Higher & $n=64(14.0 \%)$ & $n=31(12.7 \%)$ & $n=33(14.4 \%)$ \\
\hline $\begin{array}{l}\text { Previous Mental } \\
\text { Health Condition }\end{array}$ & $n=221(48.5 \%)$ & $n=104(45.8 \%)$ & $n=117(51.1 \%)$ \\
\hline $\begin{array}{l}\text { Previous Meditation } \\
\text { Experience }\end{array}$ & $n=303(66.4 \%)$ & $n=154(67.8 \%)$ & $n=149(65.1 \%)$ \\
\hline
\end{tabular}


Table 2

Descriptive Statistics of Primary and Secondary Outcome Measures, Stratified by Condition Among Protocol Adherent Participants

\begin{tabular}{lllllllll}
\hline & \multicolumn{3}{c}{$\begin{array}{c}\text { Mind-OP } \\
\end{array}$} & \multicolumn{3}{c}{$(n=68)$} & & \multicolumn{3}{c}{$\begin{array}{c}\text { Active Control } \\
(n=91)\end{array}$} \\
\cline { 2 - 10 } & \multicolumn{2}{c}{ Pre } & \multicolumn{2}{c}{ Post } & \multicolumn{2}{c}{ Pre } & Post \\
\cline { 2 - 10 } Measure & $M(S D)$ & $95 \%$ CI & $M(S D)$ & $95 \%$ CI & $M(S D)$ & $95 \%$ CI & $M(S D)$ & $95 \%$ CI \\
\hline GAD-7 & 8.82 & 7.82, & 5.06 & 3.94, & 9.71 & 8.57, & 7.66 & 6.54, \\
& $(4.16)$ & 9.83 & $(4.63)$ & 6.18 & $(5.43)$ & 10.85 & $(5.31)$ & 8.77 \\
\hline PHQ-9 & 10.13 & 8.91, & 6.24 & 4.86, & 10.91 & 9.67, & 8.08 & 6.78, \\
& $(5.05)$ & 11.35 & $(5.70)$ & 7.62 & $(5.90)$ & 12.15 & $(6.18)$ & 9.37 \\
\hline PSS & 21.78 & 20.63, & 14.01 & 12.60, & 22.56 & 21.16, & 18.56 & 16.66, \\
& $(4.73)$ & 22.93 & $(5.85)$ & 15.43 & $(6.68)$ & 23.96 & $(8.60)$ & 20.26 \\
\hline FFMQ-15 & 45.35 & 43.63 & 50.13 & 48.39, & 45.60 & 43.55, & 48.20 & 46.12, \\
& $(7.09)$ & 47.07 & $(7.22)$ & 51.88 & $(9.80)$ & 47.65 & $(9.95)$ & 50.28 \\
\hline SCS-SF & 2.57 & 2.41, & 3.10 & 2.92, & 2.52 & 2.34, & 2.79 & 2.59, \\
& $(0.65)$ & 2.73 & $(0.74)$ & 3.29 & $(0.89)$ & 2.71 & $(0.93)$ & 2.98 \\
\hline NAS-SF & 27.93 & 26.54, & 32.87 & 31.30, & 28.27 & 26.51, & 31.36 & 29.52, \\
& $(5.71)$ & 29.31 & $(6.48)$ & 34.44 & $(8.39)$ & 30.02 & $(8.76)$ & 33.19 \\
\hline TMS & 19.68 & 17.51 & 24.81 & 22.40, & 20.11 & 18.00, & 20.28 & 17.87, \\
& $(8.95)$ & 21.84 & $(9.95)$ & 27.22 & $(10.08)$ & 22.22 & $(11.49)$ & 22.58 \\
\hline Note. GAD
\end{tabular}

Note. GAD-7 = Generalized Anxiety Disorder - 7; PHQ-9 = Patient Health Questionnaire - 9; PSS = Perceived Stress Scale; FFMQ-15 = Five Facet Mindfulness Questionnaire - 15; SCS-SF $=$ Self-Compassion Scale - Short Form; NAS-SF $=$ The Nonattachment Scale - Short Form; TMS = Toronto Mindfulness Scale. 
Table 3

Intent-To-Treat (MLM) Analyses Coefficients for Main Outcome Variables

\begin{tabular}{|c|c|c|c|c|c|}
\hline & \multirow[t]{2}{*}{ Estimate } & \multirow[t]{2}{*}{ Std. Error } & \multirow[t]{2}{*}{ T-Statistic } & \multicolumn{2}{|c|}{$95 \% \mathrm{CI}$} \\
\hline & & & & Lower Bound & Upper Bound \\
\hline \multicolumn{6}{|c|}{ GAD-7 } \\
\hline Intercept & 7.81 & .45 & 17.37 & 6.92 & 8.69 \\
\hline \multicolumn{6}{|c|}{ Effect of Time $-F(5,239.10)=33.76, p<.001 *$} \\
\hline $\begin{array}{l}\text { Pre-Scores } \\
\text { (Vs. Post) }\end{array}$ & 2.28 & .41 & 5.61 & 1.48 & 3.08 \\
\hline \multicolumn{6}{|c|}{ Effect of Group $-F(1,394.81)=4.11, p=.043^{*}$} \\
\hline $\begin{array}{c}\text { Treatment } \\
\text { (Vs. Control) }\end{array}$ & -1.78 & .67 & -2.65 & -3.11 & -0.46 \\
\hline \multicolumn{6}{|c|}{ Week $*$ Group $-F(5,239)=6.70, p<.001 *$} \\
\hline \multicolumn{6}{|c|}{ PHQ-9 } \\
\hline Intercept & 8.77 & .52 & 16.78 & 7.74 & 9.80 \\
\hline \multicolumn{6}{|c|}{ Time $-F(5,235.43)=33.50, p<.001^{*}$} \\
\hline $\begin{array}{l}\text { Pre-Scores } \\
\text { (Vs. Post) }\end{array}$ & 2.79 & .45 & 6.28 & 1.92 & 3.67 \\
\hline \multicolumn{6}{|c|}{ Group $-F(1,393.23)=2.62, p=.11$} \\
\hline $\begin{array}{c}\text { Treatment } \\
\text { (Vs. Control) }\end{array}$ & -1.51 & .78 & -1.94 & -3.03 & 0.02 \\
\hline \multicolumn{6}{|c|}{ Week $*$ Group $-F(5,235.43)=3.76, p=.003^{*}$} \\
\hline \multicolumn{6}{|c|}{ PSS } \\
\hline Intercept & 18.52 & .62 & 30.03 & 17.30 & 19.73 \\
\hline \multicolumn{6}{|c|}{ Time $-F(5,231.95)=38.03, p<.001^{*}$} \\
\hline $\begin{array}{l}\text { Pre-Scores } \\
\text { (Vs. Post) }\end{array}$ & 3.89 & .55 & 7.06 & 2.80 & 4.98 \\
\hline \multicolumn{6}{|c|}{ Group $-F(1,387.08)=7.65, p=.006^{*}$} \\
\hline $\begin{array}{c}\text { Treatment } \\
\text { (Vs. Control) }\end{array}$ & -3.65 & .93 & -3.94 & -5.47 & -1.83 \\
\hline
\end{tabular}

Week $*$ Group $-F(5,231.95)=6.09, p<.001 *$

Note. GAD-7 = Generalized Anxiety Disorder - 7; PHQ-9 = Patient Health Questionnaire - 9; PSS $=$ Perceived Stress Scale.

$*$ = significant effects at $a=.05$. 
Table 4

Pre-Post T-Test Analyses and Effect Sizes on Primary and Secondary Outcomes, Stratified by Randomization Condition

\begin{tabular}{lcccccc}
\hline \multirow{2}{*}{ Measure } & \multicolumn{2}{c}{ Mind-OP Intervention } & \multicolumn{4}{c}{ Active Control Condition } \\
\cline { 2 - 7 } & Mean Diff & SD of Diff & Cohen's d & Mean Diff & SD of Diff & Cohen's d \\
\hline GAD-7 & 3.75 & 4.59 & $\mathbf{. 8 2}$ & 2.12 & 4.78 & $\mathbf{. 4 4}$ \\
PHQ-9 & 3.90 & 4.61 & $\mathbf{. 8 5}$ & 2.84 & 5.27 & $\mathbf{. 5 4}$ \\
PSS & 7.76 & 5.35 & $\mathbf{1 . 4 5}$ & 4.04 & 6.38 & $\mathbf{. 6 3}$ \\
FFMQ-15 & -4.78 & 5.67 & $\mathbf{. 8 4}$ & -2.62 & 6.28 & $\mathbf{. 4 2}$ \\
SCS-SF & -0.53 & 0.66 & $\mathbf{. 8 0}$ & -0.26 & 0.57 & $\mathbf{. 4 6}$ \\
NAS-SF & -4.94 & 5.07 & $\mathbf{. 9 7}$ & -3.03 & 6.54 & $\mathbf{. 4 6}$ \\
TMS & -5.13 & 9.02 & $\mathbf{. 5 7}$ & -0.17 & 9.11 & .02 \\
\hline
\end{tabular}

Note. Bolded effect sizes signify significant pre-post differences at $a=.05$. GAD-7 $=$

Generalized Anxiety Disorder - 7; PHQ-9 = Patient Health Questionnaire - 9; PSS = Perceived Stress Scale; FFMQ-15 = Five Facet Mindfulness Questionnaire -15 ; SCS-SF = SelfCompassion Scale - Short Form; NAS-SF $=$ The Nonattachment Scale - Short Form; TMS = Toronto Mindfulness Scale. 
Table 5

Pre-Post T-Test Analyses and Effect Sizes on Subfacets of the TMS and FFMQ-15, Stratified by Randomization Condition

\begin{tabular}{lllllll}
\hline \multirow{2}{*}{ Measure } & \multicolumn{2}{l}{ Mind-OP Intervention } & \multicolumn{3}{l}{ Active Control Condition } \\
\cline { 2 - 7 } & $\begin{array}{l}\text { Mean } \\
\text { Diff }\end{array}$ & SD & Cohen's d & Mean Diff & SD & Cohen's d \\
\hline TMS-Dec & -3.53 & 5.21 & $\mathbf{. 6 8}$ & -0.11 & 5.12 & .02 \\
TMS-Cur & -2.49 & 6.00 & $\mathbf{. 4 5}$ & -0.11 & 6.29 & .02 \\
FFMQ-Des & -0.51 & 1.70 & $\mathbf{. 3 0}$ & -0.80 & 2.26 & $\mathbf{. 3 5}$ \\
FFMQ-Obs & -0.94 & 2.09 & $\mathbf{. 4 5}$ & -0.02 & 2.18 & .01 \\
FFMQ-Aw & -1.15 & 2.10 & $\mathbf{. 5 5}$ & -0.46 & 2.13 & $\mathbf{. 2 2}$ \\
FFMQ-NJ & -1.25 & 2.48 & $\mathbf{. 5 0}$ & -0.78 & 2.64 & $\mathbf{. 3 0}$ \\
FFMQ-NR & -0.93 & 2.35 & $\mathbf{. 4 0}$ & -0.55 & 2.32 & $\mathbf{. 2 4}$ \\
\hline
\end{tabular}

Note. Bolded effect sizes signify significant pre-post changes at $a=.05$. TMS $=$ Toronto Mindfulness Scale; TMS-Dec = Decentring Subscale; TMS-Cur = Curiosity Subscale; FFMQ-15 = Five Facet Mindfulness Questionnaire - 15; FFMQ-Des = Describe Subscale; FFMQ-Obs = Observe Subscale; FFMQ-Aw = Awareness Subscale; FFMQ-NJ = Non-judge Subscale; FFMQNR $=$ Non-react Subscale. 


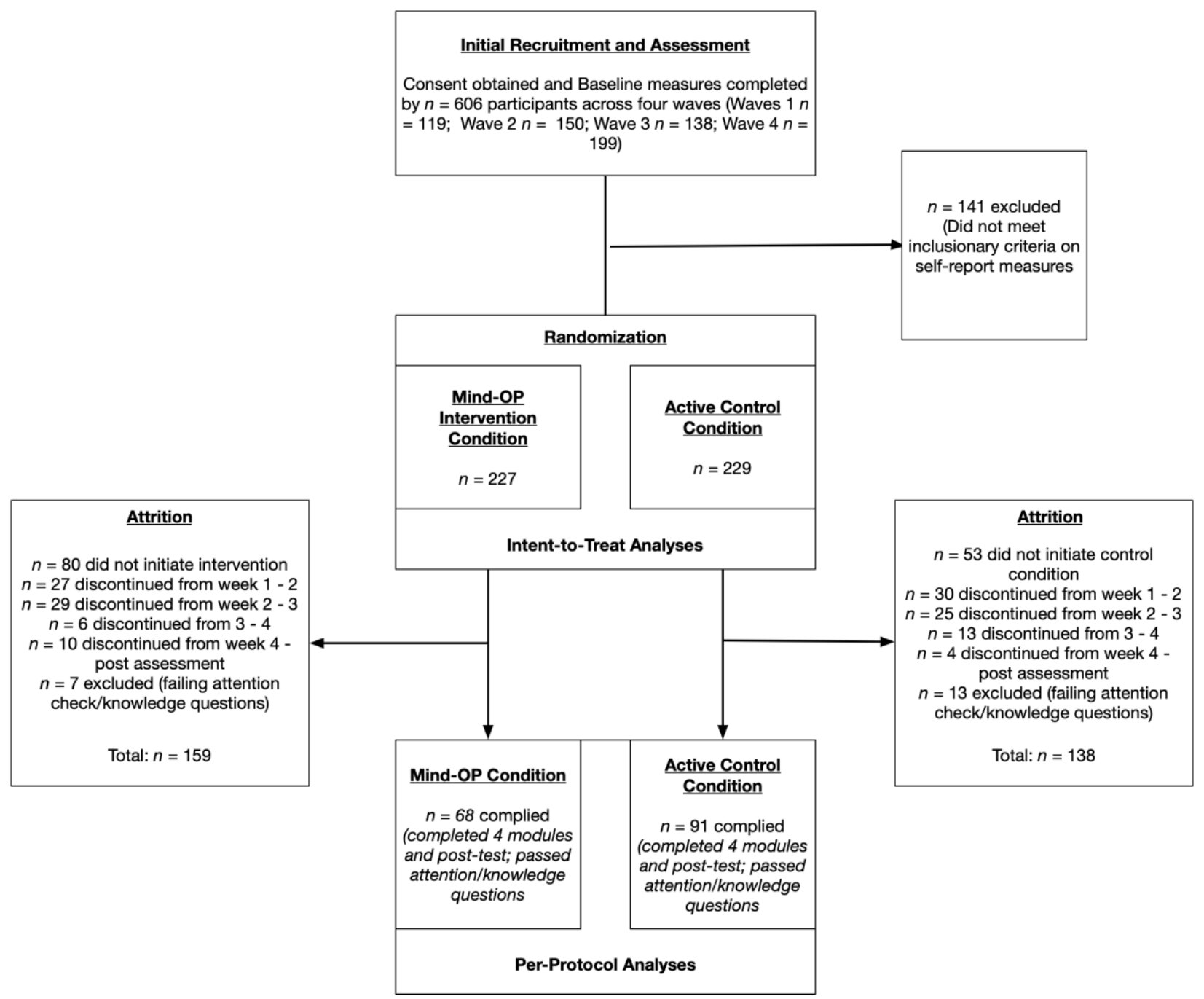

Figure 1. Participant flow throughout all timepoints in the study, stratified by randomization condition. 


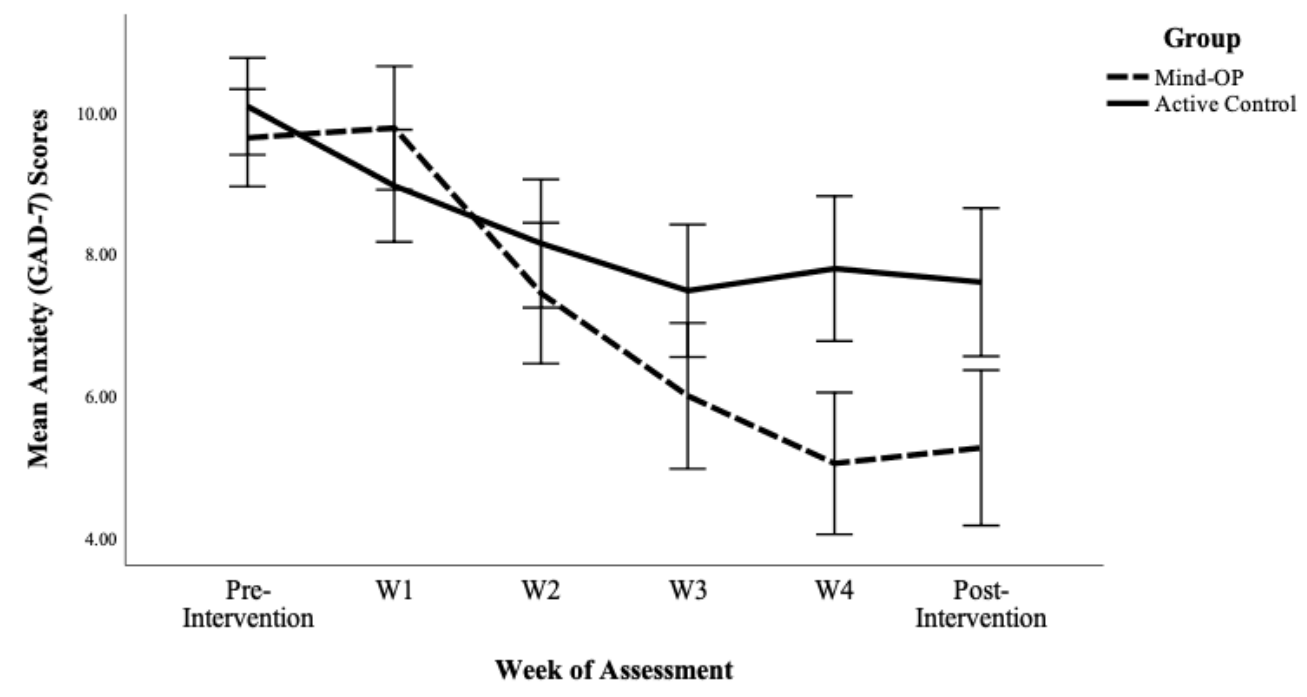

Figure 2. Mixed Linear Modeling analyses revealed significant effects of Time (week of assessment) and Group allocation on GAD-7 (Generalized Anxiety Disorder - 7) scores. 


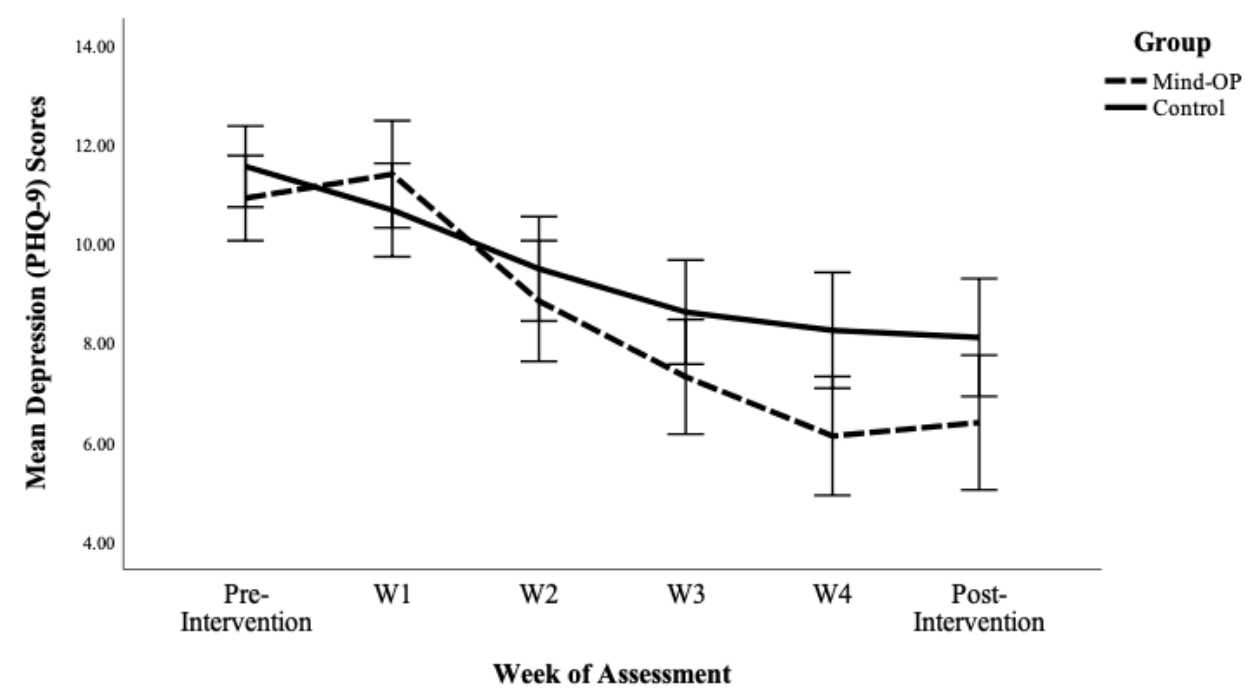

Figure 3. Mixed Linear Modeling analyses revealed significant effects of Time (week of assessment) and Group allocation on PHQ-9 (Patient Health Questionnaire - 9) scores. 


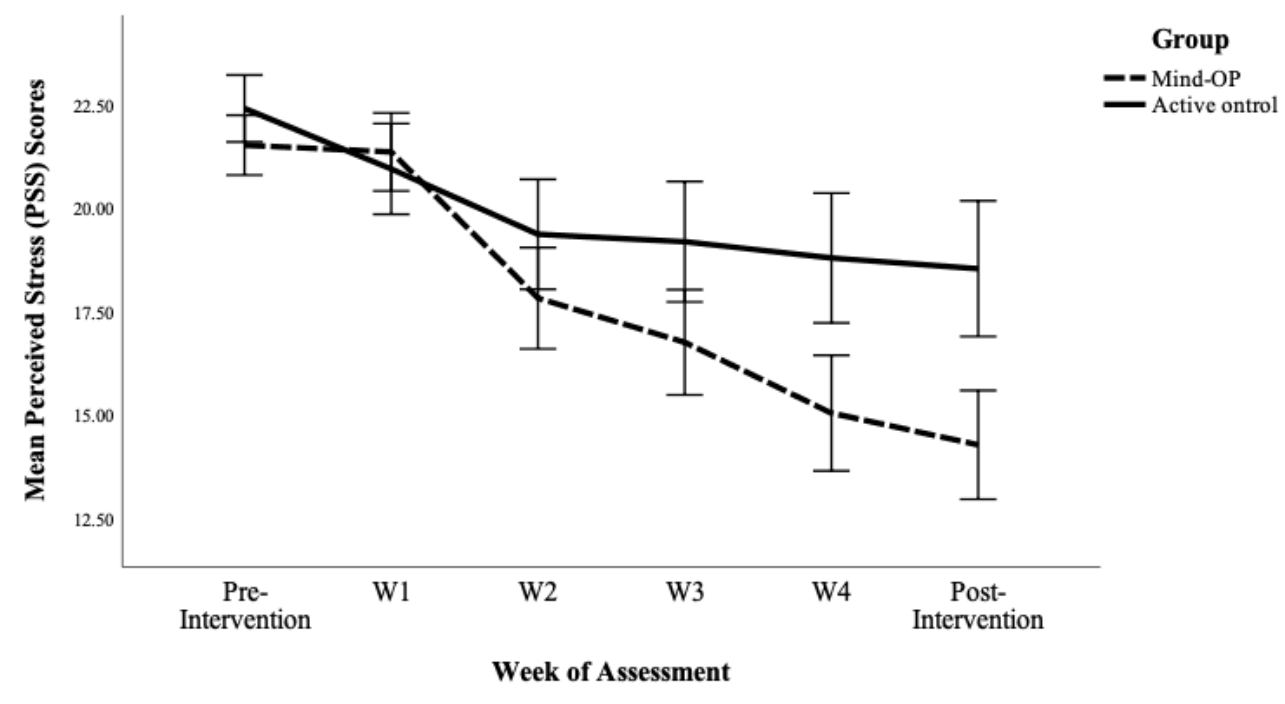

Figure 4. Mixed Linear Modeling analyses revealed significant effects of Time (week of assessment) and Group allocation on PSS (Perceived Stress Scale) scores. 